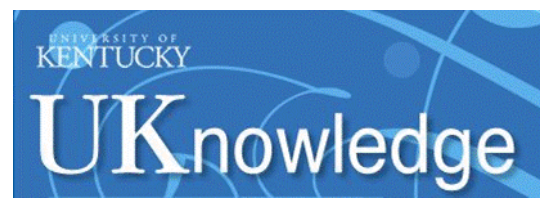

University of Kentucky

UKnowledge

\title{
Clinically Silent Alzheimer's and Vascular Pathologies Influence Brain Networks Supporting Executive Function in Healthy Older Adults
}

\author{
Brian T. Gold \\ University of Kentucky, brian.gold@uky.edu \\ Christopher A. Brown \\ University of Kentucky, christopher.a.brown@uky.edu \\ Jonathan G. Hakun \\ Pennsylvania State University \\ Leslie M. Shaw \\ University of Pennsylvania \\ John Q. Trojanowski \\ University of Pennsylvania
}

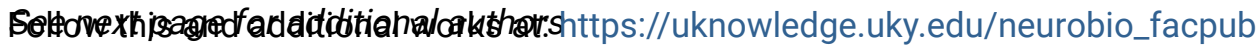

Part of the Neuroscience and Neurobiology Commons, and the Pathology Commons

Right click to open a feedback form in a new tab to let us know how this document benefits you.

\section{Repository Citation}

Gold, Brian T.; Brown, Christopher A.; Hakun, Jonathan G.; Shaw, Leslie M.; Trojanowski, John Q.; and Smith, Charles D., "Clinically Silent Alzheimer's and Vascular Pathologies Influence Brain Networks Supporting Executive Function in Healthy Older Adults" (2017). Neuroscience Faculty Publications. 56. https://uknowledge.uky.edu/neurobio_facpub/56

This Article is brought to you for free and open access by the Neuroscience at UKnowledge. It has been accepted for inclusion in Neuroscience Faculty Publications by an authorized administrator of UKnowledge. For more information, please contact UKnowledge@lsv.uky.edu. 


\title{
Clinically Silent Alzheimer's and Vascular Pathologies Influence Brain Networks Supporting Executive Function in Healthy Older Adults
}

\author{
Digital Object Identifier (DOI) \\ https://doi.org/10.1016/j.neurobiolaging.2017.06.012 \\ Notes/Citation Information \\ Published in Neurobiology of Aging, v. 58, p. 102-111. \\ (C) 2017 Elsevier Inc. All rights reserved.
}

This manuscript version is made available under the CC-BY-NC-ND 4.0 license https://creativecommons.org/licenses/by-nc-nd/4.0/.

The document available for download is the author's post-peer-review final draft of the article.

\section{Authors}

Brian T. Gold, Christopher A. Brown, Jonathan G. Hakun, Leslie M. Shaw, John Q. Trojanowski, and Charles D. Smith 


\title{
Clinically Silent Alzheimer's and Vascular Pathologies Influence Brain Networks Supporting Executive Function in Healthy Older Adults
}

\author{
Brian T Gold ${ }^{\mathrm{a}, \mathrm{c}, \mathrm{d}}$, Christopher A Brown ${ }^{\mathrm{a}}$, Jonathan G Hakun ${ }^{\mathrm{a}, 1}$, Leslie M Shaw ${ }^{\mathrm{f}}$, John $^{\mathbf{Q}}$ \\ Trojanowski ${ }^{\dagger}$, and Charles D Smith ${ }^{b, c, d}$ \\ aDepartment of Neuroscience, University of Kentucky, 800 Rose Street, Lexington, KY, 40536, \\ USA
}

bDepartment of Neurology, University of Kentucky, 800 Rose Street, Lexington, KY, 40536, USA

'Sanders-Brown Center on Aging, University of Kentucky, 800 Rose Street, Lexington, KY, 40536, USA

dMagnetic Resonance Imaging and Spectroscopy Center, University of Kentucky, 800 Rose

Street, Lexington, KY, 40536, USA

fDepartment of Pathology \& Laboratory Medicine, Institute on Aging, Center for

Neurodegenerative Disease Research, University of Pennsylvania School of Medicine,

Philadelphia, PA, USA

\begin{abstract}
Aging is associated with declines in executive function. We examined how executive functional brain systems are influenced by clinically silent Alzheimer's disease (AD) pathology and cerebral white matter hyperintensities (WMHs). Twenty-nine younger adults and thirty-four cognitively normal older adults completed a working memory paradigm while functional magnetic resonance imaging (fMRI) was performed. Older adults further underwent lumbar cerebrospinal fluid (CSF) draw for assessment of AD pathology and FLAIR imaging for assessment of WMHs. Accurate working memory performance in both age groups was associated with high fronto-visual functional connectivity (fC). However, in older adults, higher expression of fronto-visual fC was linked with lower levels of clinically silent AD pathology. In addition, AD pathology and WMHs were each independently related to increased fMRI response in the left dorsolateral prefrontal
\end{abstract}

Corresponding Author: Brian T Gold, Department of Neuroscience, University of Kentucky, Lexington, KY, 40536, USA, (859) 323-4813, brian.gold@uky.edu.

${ }_{1}^{1}$ Permanent Address: Department of Psychology, Pennsylvania State University, University Park, PA, USA

Publisher's Disclaimer: This is a PDF file of an unedited manuscript that has been accepted for publication. As a service to our customers we are providing this early version of the manuscript. The manuscript will undergo copyediting, typesetting, and review of the resulting proof before it is published in its final citable form. Please note that during the production process errors may be discovered which could affect the content, and all legal disclaimers that apply to the journal pertain.

Verification

The authors declare no conflict of interest.

The data contained in this manuscript has not been previously published, has not been submitted elsewhere and will not be submitted elsewhere while under consideration at Neurobiology of Aging.

Appropriate approval and procedures were used concerning human subjects.

All the authors have reviewed the contents of the manuscript and approved its contents as well as validate the accuracy of the data. 
cortex, a pattern associated with slower task performance. Our results suggest that clinically silent $\mathrm{AD}$ pathology is related to lower expression of a fronto-visual fC pattern supporting executive task performance. Further, our findings suggest that AD pathology and WMHs appear to be linked with ineffective increases in frontal response in $\mathrm{CN}$ older adults.

\section{Keywords}

Executive; Aging; Connectivity; Alzheimer's; Hyperintensities

\section{Introduction}

Executive function underlies the capacity for flexible, adaptive thought processes (Miller and Cohen, 2001). Executive functions are deployed when the cognitive system faces new situations, is required to temporarily maintain information, or must overcome habitual responses (Miyake et al., 2000). Aging is associated with marked decline in executive function (Schaie, 1996), which represents a strong predictor of functional independence in older adults (Bell-McGinty et al., 2002). Increased understanding of the neural underpinnings of age-related executive declines is thus an important goal for the field of aging neuroscience.

Of particular relevance are those functional brain alterations that are directly linked with executive task performance. For example, while older adults can show a variety of differences in the strength or extent of activation compared to younger adults, these differences are not always associated with task performance (Grady, 2012). Commonly reported age-related activation - performance alterations have been reported in prefrontal cortex response and in functional connectivity (fC) patterns between prefrontal cortex and posterior regions (Drag and Bieliauskas, 2010). In general, alterations in executive brain systems and performance in $\mathrm{CN}$ older adults are typically attributed to processes of normal brain aging (reviewed in Harada et al., 2013).

However, recent reviews highlight the need for increased understanding about contributions of clinically silent brain pathology to executive and other cognitive declines (Jagust, 2013). Two of the most common forms of brain pathology in CN older adults are cerebrovascular damage reflected as white matter hyperintensities (WMHs) on FLAIR images (Breteler et al., 1994) and Alzheimer's disease (AD) pathology (Shaw et al., 2009). Clinically silent vascular and $\mathrm{AD}$ pathologies may disrupt executive functional brain networks prior to clinical impairment given that executive declines represent a common feature of early/ preclinical cerebrovascular disease (Reed et al., 2004) and AD (Blacker et al., 2007).

Separate studies conducted with $\mathrm{CN}$ older adults have reported functional brain alterations during the performance of executive tasks associated with WMHs (Nordahl et al., 2006; Venkatraman et al., 2010) and more recently with AD pathology (Gordon et al., 2015; Oh et al., 2015). However, few if any studies have explored the impact of both WMH and AD pathology on functional brain activations using a common task paradigm and participant group. Here we explore this issue with a particular emphasis on identifying those alterations in functional activation patterns that directly affect executive task performance. 
Toward that end, we focused on activation patterns within fronto-visual regions linked with executive performance in our recent work (Hakun et al., 2015; Zhu et al., 2015). The previous studies used a task switching paradigm and focused on both local activation patterns in prefrontal regions and $\mathrm{fC}$ patterns between prefrontal and visual association areas. Results indicated that higher fC between the left dorsolateral prefrontal cortex (IDLPFC) and bilateral portions of the lateral occipital cortex (LOC) was associated with better executive task performance in $\mathrm{CN}$ older adults, while higher prefrontal response magnitudes were associated with poorer performance.

In the present study, a working memory fMRI paradigm was employed and associations with fronto-visual activation and $\mathrm{fC}$ patterns and performance were explored in both younger and older adult groups. We then explored the separate and potentially interactive relationships between WMHs and AD pathology on those functional activation patterns linked with executive performance in older adults. We expected to replicate our previous findings of fronto-visual activation-performance relationships. In addition, based on our previous results and the literature, we hypothesized that clinically silent AD pathology and/or WMH may contribute to weaker IDLPFC-LOC fC and higher local IDLPFC response magnitude.

\section{Materials and Methods}

\subsection{Participants}

Sixty-eight participants (38 older adults and 30 younger adults) were initially recruited. Written informed consent was obtained from each participant under an approved University of Kentucky (UK) Institutional Review Board protocol. Younger adults were recruited from the university and surrounding community through advertisements. Older adults were recruited from an existing cohort of volunteers at the Sanders-Brown Center on Aging (SBCoA), part of UK's Alzheimer's Disease Center (ADC). Participants at the SBCoA are generally healthy, cognitively normal, and live independently in the greater Lexington, Kentucky area (Schmitt et al., 2012).

Older adult participants completed the Uniform Data Set (UDS2) used by US ADCs supported by the NIH National Institute on Aging (NIA/NIH ADCs) (Morris et al., 2006). The UDS includes a detailed medical history, physical examination, assessment of behavioral symptoms, global assessment with the Clinical Dementia Rating (CDR), and a neuropsychological battery (Weintraub et al., 2009). The neuropsychological battery includes tests related to the domains of episodic memory, executive function, speed of processing, language and visuospatial function. Participants' results are evaluated by the ADC clinical core and a clinical consensus diagnosis is reached. All older adult participants in the present study had a clinical consensus diagnosis of 'cognitive normal' and a CDR global score $=0$.

Exclusionary criteria for the present study were a significant head injury (operationally defined as a loss of consciousness greater than five minutes), the use of psychotropic medication, claustrophobia, pacemakers, or the presence of metal implants contraindicated for MRI. Subsequent quality control measures led to the exclusion of one younger adult and four older adults from results reported here. The younger adult was excluded due to a large 
artefact on their MR images. The four older adults were excluded due to: excessive motion during MRI scanning (one participant); task accuracy or response rate $\geq 3$ standard deviations below their group mean ( 3 participants). Characteristics of the final group participants (29 younger adults 34 older adults) are shown in Table 1.

\subsection{Task Design}

Participants performed a modified version of the delayed-match to sample working memory task (Figure 1) (Jiang, 2000). The task was designed to challenge executive components of working memory via requirements to maintain multiple target stimuli active in mind over an extended delay interval, during which time distractor trials (non-match images) can be new or repeated (Kane and Engle, 2002). The task stimuli were two-dimensional pictures of common objects selected from Snodgrass and Vanderwart database (Snodgrass and Vanderwart, 1980). During task blocks, participants were asked to 'hold in mind' the two target images and indicate (via button press) whether or not each of 12 serially presented single samples represented a match with either target image. Sample images were either one of the two target (match) images presented at the beginning of the task block or distractor (non-match) images, which were repeated between 2-4 times in a block. The ratio of targets to distractors in each block ranged from 5:7 to 7:5. During baseline blocks, participants viewed scrambled versions of object images (created by Fast Fourier transform algorithms) and pressed response buttons to each image to control for basic visuomotor components of the task.

The experiment was divided into two fMRI runs. Each run consisted of 8 task blocks ( 28 sec/per) interleaved with and 8 visuomotor baseline blocks (10 sec/per). Responses were made using MRI compatible hand-held response button boxes, with button press response mappings counterbalanced across participants. Stimuli were presented using E-prime software (Psychology Software Tools, Inc., Pittsburgh, PA) and projected to a screen using an MRI compatible projector. Participants viewed the screen via a mirror mounted on the MRI head coil. The stimulus presentation program was used to log response time and accuracy for each working memory trial. Participants completed a brief practice session prior to fMRI scanning.

\subsection{Behavioral Analysis}

Accuracy was measured using the corrected recognition (CR) rate, computed by subtracting the false alarm rate ( $\%$ of non-match trials incorrectly identified as targets) from the hit rate (\% of match trials correctly identified as targets). Mean reaction time (RT) was calculated across responses.

\subsection{MRI Acquisition}

Neuroimaging was performed using a Siemens TIM Trio 3 Tesla scanner with a 32-channel head coil at the UK's Magnetic Resonance Imaging and Spectroscopy Center. Two highresolution T1-weighted anatomic images were collected using a magnetization-prepared rapid acquisition with gradient echo (MPRAGE) sequence [repetition time $(\mathrm{TR})=2530 \mathrm{~ms}$, echo time $(\mathrm{TE})=2.26 \mathrm{~ms}$, inversion time $(\mathrm{TI})=1100 \mathrm{~ms}$, flip angle $(\mathrm{FA})=7^{\circ}$, acquisition matrix $=256256176$, field of view $(\mathrm{FOV})=256 \mathrm{~mm}^{2}$, isotropic $1 \mathrm{~mm}$ voxels $]$. Blood- 
oxygen-level dependent (BOLD) T2*-weighted scans were acquired with a gradient-echo planar imaging (EPI) sequence $\left[\mathrm{TR}=2000 \mathrm{~ms}, \mathrm{TE}=27 \mathrm{~ms}, \mathrm{FA}=83^{\circ}, \mathrm{FOV}=224 \mathrm{~mm}^{2}\right.$, acquisition matrix $=6464$, resolution $=3.8 \mathrm{~mm}$ isotropic, 36 interleaved slices]. Fluidattenuated inversion recovery (FLAIR) images were acquired from older adults with a fat saturated turbo-spin echo $(\mathrm{TSE})$ sequence $[\mathrm{TR}=9000 \mathrm{~ms}, \mathrm{TE}=89 \mathrm{~ms}, \mathrm{TI}=2500 \mathrm{~ms}, \mathrm{FA}=$ $130^{\circ}$, acquisition matrix $=256 \times 174 \times 34,1 \mathrm{~mm} \times 1 \mathrm{~mm} \times 4 \mathrm{~mm}$ voxels $]$.

\section{5. fMRI Preprocessing and Analyses}

The FMRIB Software Library [FSL, (Smith et al., 2004; Jenkinson et al., 2012)] was used for fMRI data preprocessing and analyses. The T1-weighted structural image collected closest in time to the fMRI scans was bias-field corrected using FAST and brain extracted using BET. fMRI data were motion corrected, smoothed with a $9 \mathrm{~mm}$ Gaussian filter, and high-pass filtered at 128s using FEAT v.6.0.0. Participants with $>1 \mathrm{~mm}$ root mean square absolute motion were excluded from further processing (one older adult). The remaining participants' functional data were registered to the high-resolution T1-weighted images using FLIRT. T1-weighted images were then registered to MNI space and resampled to $2 \mathrm{~mm}$ isotropic voxels using FLIRT to generate an initial linear affine transformation, followed by non-linear warping using FNIRT with 20mm warp resolution. Participants' non-linear warp parameters were then applied to their functional data, which was resampled to MNI $2 \mathrm{~mm}$ space.

fMRI Hypothesis-Driven Analyses-A priori, hypothesis-driven analyses were conducted focusing on the left dorsolateral prefrontal cortex (IDLPFC) and the left and right lateral occipital cortices (ILOC, rLOC). These regions were selected for a priori analyses based on results from one of our recent studies (described in the Introduction) involving an independent data set consisting of 65 participants (32 younger versus 33 older adults) using a different visual executive task (a switching paradigm) (Hakun et al., 2015). In the present experiment, ROI masks ( $6 \mathrm{~mm}$ spheres) were generated to surround peak MNI coordinates from results on the present data set to maximize sensitivity of mask locations to the current participant sample and task paradigm. Specifically, the ROI masks were generated on results from an independent components analysis (ICA) to ensure that neither age group contributed disproportionally to mask seed locations.

Tensor-based ICA used FSL's MELODIC (Beckmann and Smith, 2005), with automatic determination of the number of components to extract. In addition, a model of the task paradigm was included in the ICA analysis to test whether the main ICA component reflected a general task-related (i.e. working memory) response functional pattern. ROI masks (6 mm spheres) were generated to surround MNI peaks associated with the main ICA component. Time series data were then extracted within each ROI, from each participant's pre-processed fMRI data. Data extracted from the three ROIs were then submitted to BOLD magnitude and functional connectivity (fC) analyses.

fMRI BOLD Magnitude Analysis-BOLD magnitudes were extracted from the time series data for each participant/ROI/block. Mean BOLD signal was computed across TRs corresponding to the maintenance/decision epoch of the working memory task. The TR 
immediately before each task block onset was selected as a baseline. Mean percent signal change between baseline and task was then computed for each participant/ROI across task blocks within each run and then averaged across runs.

fMRI Functional Connectivity (fC) Analysis-A modified beta-series fC analysis was performed (Rissman et al., 2004). Each participant's mean task-related BOLD signals for each ROI/block (described above) were Z-scored within each of their two runs. The Z-scores from the two runs were then concatenated to form a single beta-series consisting of 16 values ( 1 per task block) for each participant/ROI that reflected the fluctuation around the mean of the maintenance/decision epoch of each block. Zero-order correlations were performed between the IDLPFC and ILOC beta-series and between the IDLPFC and rLOC beta-series in order to determine the interregional coherence between regions over task blocks. The resulting correlation coefficients were used as the measure of $\mathrm{fC}$ between ROIs.

\subsection{WMH Assessment Procedures}

WMH volumes were computed in older adults using an overall framework employed in our recent work (Smith et al., 2016). The series of steps included field correction using the N3 algorithm, co-registration of the two T1-weighted images using FSL's Linear Registration Tool (FLIRT), T1 image averaging, skull-stripping and segmenting using Freesurfer. Subsequent manual editing was performed to remove artifacts particularly at the base of the brain, between the lateral ventricles and at the level of the superior sagittal sinus. The skullstripped T1 image was then registered to the FLAIR image using FLIRT. A white matter mask was generated from the segmentation results from Freesurfer by combining left cortical WM, right cortical WM, and WM hypo-intensities. This WM mask was registered to the FLAIR image using the same affine transformation generated above.

The WM mask was applied to the FLAIR image, and mean and standard deviation of WM signal intensities were estimated based on voxel histogram fitting using a 2-gaussian model. The voxel intensity histogram was thresholded at a standard deviation of 2.33 from the mean of the dominant (normal appearing white matter) gaussian fit. The volume of hyperintensities exceeding the threshold was recorded for each participant as whole-brain (total) WMH volume. In addition, a separate measure of frontal WMH volume was computed due to the importance of the frontal lobes to executive function. The frontal WMH measure was computed by applying the MNI Structural Atlas Frontal Lobe Mask, which was edited to include white matter. Participants' WMH volumes were corrected for their total intracranial volume (ICV).

\subsection{CSF Collection and Analysis}

Older adults underwent lumbar CSF draw in the morning following an overnight fast using a protocol previously described (Gold et al., 2014). CSF draw was performed within one week of MRI scanning. One set of aliquots were shipped on dry ice from the University of Kentucky to the Biomarker Research Laboratory at the University of Pennsylvania Medical Center. Samples were kept frozen until being thawed just prior to analysis. CSF analysis was performed following the standard Alzheimer's Disease Neuroimaging Initiative protocol as 
previously described (Shaw et al., 2009). CSF concentrations of $A \beta_{42}$, total tau (t-tau), and phosphorylated tau $(\mathrm{p}$-tau 181$)$ were measured.

\subsection{Statistical Analyses}

Statistical analyses were performed using SPSS 22 (IBM, Chicago, IL). Potential betweengroup differences in task behavioral measures (CR and RT) and fMRI patterns (IDLPFC magnitude and IDLPFC-LOC functional connectivity) were explored using independent samples t-tests. All within-group analyses used multiple linear regression with age, sex and education as covariates. For RT, outliers were identified as values $\geq 3$ standard deviations from their group mean. This criterion resulted in the removal of between $2.9 \%$ and $5.7 \%$ of total data points from regression analyses. Separate regression models tested possible relationships between task performance and fMRI patterns in younger and older groups. Additional regression models in the older group were run to explore the possible influence of measures of pathology (CSF markers, or WMHs) on functional measures (fMRI or performance measures). CSF and WMH values were log-transformed before statistical analyses.

\section{Results}

\subsection{Demographic and Behavioral Results}

Group demographic and behavioral scores are shown in Table 1. There were no group differences in male/female ratio $\left(\chi^{2}=0.10, d f=1, p=0.75\right)$ or years of education $(t=0.09$, $d f=61, p=0.93$ ). Mean task accuracy was high for both the younger adult group [mean CR $=94.1 \%$ (mean hit rate $=97.1 \%$, mean false alarm rate $=2.96 \%)$ and the older adult group $[($ mean $\mathrm{CR}=86.1 \%$ (mean hit rate $=90.5 \%$, mean false alarm rate $=4.41 \%)]$. Older adults had significantly lower accuracy $(t=-4.20, d f=61, p<.001)$ and higher RT $(t=8.89, d f=$ $61, p<.001)$ than younger adults. Further, accuracy and RT were inversely correlated across participants $[r(61)=-0.45 p<.001]$.

\subsection{ICA Results}

ICA generated 27 independent components (IC), explaining $83.3 \%$ of the total variance in the data. The selected IC (Figure 2, A-D and Table 2) included regions often activated during working memory tasks, including the IDLPFC, frontal eye fields, anterior insula, anterior cingulate, PPC and LOC. This IC was significant for the task model $\left(F_{3,151}=48.5\right.$, $p<.001)$.

\subsection{ROI Results}

ROIs were generated to surround peak coordinates of the IDLPFC, ILOC, and rLOC from the main IC (Table 2). There were no lateralization differences in BOLD magnitude or fC values in either age group ( $p$ 's $\geq 0.30$ ). Left and right LOC BOLD magnitudes were thus combined into a single BOLD magnitude LOC measure and $\mathrm{fC}$ measures were combined into a single IDLPFC-LOC fC measure. There were no between-group differences in BOLD magnitudes in LOC $(t=-0.28, d f=61, p=0.80)$ or in BOLD magnitudes in the IDLPFC $(t$ $=0.29, d f=61, p=0.77)$. There were no between-group differences in IDLPFC-LOC fC $(t$ $=-0.51, d f=60, p=0.61)$ (Figure 3E). 


\subsection{Relationship between Functional Patterns and Task Performance}

Within the younger group, DLPFC-LOC fC was positively associated with accuracy ( $\beta=$ $0.42, d f=24, \mathrm{p}=0.05$; Figure 3A) but not with RT ( $\beta=-0.19, d f=24, \mathrm{p}=0.43)$, while IDLPFC magnitude was not associated with accuracy $(\mathrm{p}=0.51)$ or $\mathrm{RT}(\mathrm{p}=0.84$ Figure $3 \mathrm{~B})$. Within the older group, IDLPFC-LOC fC was positively associated with accuracy ( $\beta=0.39$, $d f=28, \mathrm{p}=0.03$; Figure $3 \mathrm{C}$ ) but not RT $(\beta=-0.11, d f=28, \mathrm{p}=0.57)$, while 1DLPFC BOLD magnitude was not associated with accuracy $(\beta=-0.094, d f=28, \mathrm{p}=0.60)$ but was positively associated with RT $(\beta=0.48, d f=28, \mathrm{p}=.005$; Figure $3 \mathrm{D})$.

\subsection{Relationship between Clinically Silent Pathologies}

Mean values for CSF markers and WMHs in the older adult group are shown in Table 3, and are similar to values reported in other cohorts of cognitively normal older adults (Shaw et al., 2009; Venkatraman et al., 2010). Higher WMH values and CSF values (including CSF ratios) indicate higher pathology, except for $\mathrm{CSF} A \beta_{42}$, where higher values indicate lower pathology. There were no significant relationships between any of the CSF markers with either total WMH or frontal WMHs (all p's $\geq 0.13$ ).

\subsection{Clinically Silent Pathologies and IDLPFC-LOC fC Scores}

Separate multiple linear regression models were run with each CSF marker $\left(A \beta_{42}, t-t a u, p-\right.$ $\operatorname{tau}_{181}$ and the $\mathrm{t}$-tau/A $\beta_{42}$ ratio) as a predictor of IDLPFC-LOC fC scores. Results indicated that the $\mathrm{t}$-tau $/ \mathrm{A} \beta_{42}$ ratio was a significant predictor $(\beta=-0.36, d f=28, p=0.04$; Figure 4$)$, whereas none of the other CSF markers were predictors of IDLPFC-LOC fC scores (all $p$ 's $\geq$ 0.33 ). Separate multiple linear regression models run with each measure of WMH volume indicated that neither total WMH volume ( $p=0.13$ ), nor with frontal WMH volume ( $p=$ $0.19)$, were predictors of IDLPFC-LOC fC scores.

Based on these results, two further models were run to determine if effects of the t-tau/A $\beta_{42}$ ratio on IDLPFC-LOC fC scores were independent of WMH volumes. Results indicated the $\mathrm{t}$-tau/A $\beta_{42}$ ratio remained a significant predictor of DLPFC-LOC fC scores in the model including total $\mathrm{WMH}$ volume as a predictor $(\beta=-0.37, \mathrm{df}=28, \mathrm{p}=0.03)$ and in the model that included frontal WMH volume as a predictor $(\beta=-0.34, \mathrm{df}=28, \mathrm{p}=0.05)$.

Two final models were run to test for possible interactions. No interactions were found between the t-tau/A $\beta_{42}$ ratio and total WMH volume or frontal WMH volume on IDLPFCLOC fC scores ( $p$ 's $\geq 0.33)$.

\subsection{Clinically Silent Pathologies and IDLPFC Magnitude}

Separate multiple linear regression models were run with each CSF marker as a predictor of 1DLPFC magnitudes. Results indicated that $A \beta_{42}$ was a significant predictor of IDLPFC magnitudes $(\beta=-0.41, d f=29, p=0.02$; Figure $5 \mathrm{~A}), \mathrm{t}$-tau $/ \mathrm{A} \beta_{42}$ ratio was a marginally significant predictor $(\beta=0.34, d f=28, p=0.051)$, while the other CSF markers were not predictors of IDLPFC magnitudes (both $p$ 's $\geq 0.45$ ). Separate multiple linear regression models run with each measure of WMH volume indicated that frontal WMH volume was a significant predictor of IDLPFC magnitudes ( $\beta=0.36, \mathrm{df}=28, \mathrm{p}=0.047$; Figure $5 \mathrm{~B}$ ), but total WMH volume was not $(\mathrm{p}=0.36)$. 
Based on these results, a model was run to determine if the effects of $A \beta_{42}$ and frontal WMH volumes on IDLPFC magnitude were independent or shared. Results of the model including both forms of pathology indicated that $\mathrm{A} \beta_{42}(\beta=-0.37, \mathrm{df}=28, \mathrm{p}=0.04)$ and frontal WMH volume $(\beta=0.33, \mathrm{df}=28, \mathrm{p}=0.049)$ were independent predictors of IDLPFC magnitude.

A final model was run to test for a possible interaction but indicated no interaction between $\mathrm{A} \beta 42$ and frontal WMH volumes on IDLPFC magnitude $(\mathrm{p}=0.23)$.

\subsection{Clinically Silent Pathologies and Task Performance}

The $\mathrm{t}$-tau $/ \mathrm{A} \beta_{42}$ ratio was positively associated with $\mathrm{RT}(\beta=0.41, d f=27, p=0.02)$ and showed a trend toward a negative relationship with accuracy $(\beta=-0.31, d f=28, p=0.067)$. $\mathrm{A} \beta_{42}$ was negatively associated with $\mathrm{RT}(\beta=-0.48, d f=28, p=0.003)$ but not accuracy ( $p$ $=0.16$ ). None of the other CSF markers showed a relationship with performance measures (all $p$ 's 20.31 ). Neither total WMH volume nor frontal WMH volumes were associated with performance measures (all $p$ 's $\geq 0.12$ ).

\section{Discussion}

We explored the effects of age, AD pathology and WMHs on brain activation patterns associated with working memory task performance. Results indicated that fronto-visual fC strength was positively associated with working memory accuracy in both younger adults and $\mathrm{CN}$ older adults. In contrast, high IDLPFC response was associated with slower RT, but only in older adults. Further, within older adults, both IDLPFC and fronto-visual fC activation patterns were linked with levels of clinically silent pathology. Specifically, our results indicate that higher levels of AD pathology are associated with lower fronto-visual fC and suggest that levels AD pathology and WMHs may be independently related to DLPFC response in $\mathrm{CN}$ older adults.

\subsection{Age-Related Neurocognitive Patterns}

We employed a task designed to challenge executive components of working memory via the use of an extended delay interval and introduction of distractor trials. Both older and younger groups performed the working memory task with high accuracy (younger mean = 94.7\%; older mean $=86.1 \%$ ). However, older adults performed less accurately and slower than younger adults, a finding consistent with the working memory literature (Sander et al., 2012), particularly for tasks in which interference is high (Hedden and Park, 2003). Also consistent with previous findings, working memory task performance was associated with activation of a distributed cortical network including the DLPFC, frontal eye fields, anterior insula, anterior cingulate, posterior parietal cortex and LOC regions (Niendam et al., 2012). Within this network of regions, we explored fronto-visual activation patterns previously linked with executive task performance in our recent work (Hakun et al., 2015; Zhu et al., 2015). 


\subsection{Age-Invariant Fronto-Visual fC - Performance Relationships}

Our results showed that high fronto-visual (IDLPFC-LOC) fC was positively associated with working memory performance in both younger and older adults. The age-invariant performance boost associated with high IDLPFC-LOC fC did not reflect a speed-accuracy tradeoff, as accuracy and RT were inversely correlated in our study. Further, our findings showed that connectivity between fronto-visual regions was associated with accuracy, but not RT, in both younger and older adults. These findings suggest that coordinated functioning between fronto-visual regions appears to aid working memory accuracy without sacrificing speed.

The DLPFC contributes to multiple cognitive control processes (Niendam et al., 2012) and flexibly interacts with domain-preferential (i.e. object/spatial/verbal) posterior brain regions during working memory tasks (Gazzaley and Nobre, 2012). The LOC supports visual object representation, including non-spatial visual features and object identity (Grill-Spector et al., 2001). High IDLPFC-LOC fC during our task may thus relate to top-down biasing of attentional processes to facilitate target-distractor discrimination during the maintenance/ decision epoch, consistent with our previous findings in the domain of task switching (Hakun, et al., 2015).

\subsection{Fronto-Visual $\mathrm{fC}$ and Clinically Silent $\mathrm{AD}$ Pathology}

Our results indicated that higher levels of $\mathrm{AD}$ pathology were associated with lower expression of the DLPFC-LOC fC pattern supporting working memory performance. A recent PIB-PET study reported negative effects of amyloid on medial temporal fC patterns during an episodic memory encoding task (Oh and Jagust, 2013). Our results extend these findings by showing that $\mathrm{AD}$ pathology was associated with lower expression of an $\mathrm{fC}$ pattern that was linked with better in-scanner executive task performance. Specifically, we found that IDLPFC-LOC fC was negatively correlated with the t-tau/A $\beta_{42}$ ratio (but not with tau or $\mathrm{A} \beta_{42}$ individually).

We interpret this finding as reflective of the increased sensitivity of the t-tau/A $\beta_{42}$ ratio to the presence of preclinical $\mathrm{AD}$ relative to tau or $\mathrm{A} \beta_{42}$ individually (Shaw et al., 2009). At the behavioral level, previous results have reported that the $t-\operatorname{tau} / \mathrm{A} \beta_{42}$ ratio is more predictive of imminent cognitive decline in $\mathrm{CN}$ older adults than either t-tau or A $\beta 42$ individually (Fagan et al., 2007; Vos et al., 2013). Consistent with this possibility, our results indicated a trend for a negative association between working memory accuracy and the tau/A $\beta_{42}$ ratio in $\mathrm{CN}$ older adults, but not for either tau or $A \beta_{42}$.

The mechanisms through which tau and amyloid may synergistically disrupt fC are likely to be multifaceted and may include deleterious effects on synaptic signaling. For example, $\mathrm{A} \beta_{42}$ has been shown to aggregate at synapses (Lazarov et al., 2002), which may impair synaptic functions (Hardy and Selkoe, 2002). Tau provides structural support to axonal microtubules, and loss of this function disrupts normal axonal functioning (Iqbal et al., 2009). Our finding that AD pathology was negatively associated with fC in portions of an organized functional network appears consistent with notion that $\mathrm{AD}$ lesions may propagate trans-synaptically across interconnected regions (Pearson and Powell, 1989). 


\subsection{DLPFC Response, Clinically Silent AD Pathology, and WMHs}

We found that AD pathology and frontal WMHs were associated with higher IDLPFC response magnitude. Several previous studies have also observed positive relationships between AD pathology and BOLD response during episodic memory tasks (Sperling et al., 2009; Mormino et al., 2012; Elman et al., 2014) and executive tasks (Gordon et al., 2015; Oh et al., 2015). Considering WMH-BOLD relationships in CN older adults, there have been reports of both positive (Lockhart et al., 2015) and negative correlations (Nordahl et al., 2006; Venkatraman et al., 2010). There is no clear consensus in the literature if increased BOLD activation in response to cerebral pathology represents successful compensation (Elman et al, 2014), a failed attempt at compensation (Oh et al., 2013), or merely an aberrant response to neurodegenerative processes (Lockhart et al., 2015). Our findings appear more consistent with the latter two possibilities as IDLPFC magnitude was positively associated with both AD pathology and WMHs and yet was also linked with slower RT.

A question that arises from this literature is whether the effects of $\mathrm{AD}$ and vascular pathologies on functional brain response patterns are independent or synergistic. Increasing evidence suggests that amyloid accrual and WMHs become linked over time and exacerbate each other (Brickman et al., 2015; Scott et al., 2016). For example, amyloid accrual within blood vessels (i.e. cerebral amyloid angiopathy) can exacerbate small vessel injury processes that contribute to WMHs (Gurol et al., 2013). In addition, small vessel disease may reduce clearance of amyloid leading to greater amyloid accrual (Zlokovic, 2005). Nevertheless, cross-sectional results in $\mathrm{CN}$ older adults remain mixed, with some studies finding relationships between AD pathology and WMHs (Scott et al., 2015; Marnane et al., 2016) and others, such as the present study, observing non-significant relationships (Lo and Jagust, 2012; Barnes et al., 2013).

Moreover, little remains known about the relative dependence/independence of $\mathrm{AD}$ pathology and WMHs on fMRI patterns. By obtaining measures of AD pathology, WMHs and task-related fMRI on a common participant group, we were able show that $\mathrm{A} \beta$ and WMH were unique predictors of IDLPFC response in CN older adults. This suggests that $A \beta$ and WMH may influence brain functional response through different mechanisms. For example, $A \beta$ may induce aberrant neural response (Palop and Mucke, 2010) which, in turn, may contribute to increased $A \beta$ release (Cirrito et al., 2005). In contrast, WMH are associated with diffuse disconnection of WM tracts (Mayda et al., 2011). A finding of higher BOLD response associated with lower WM connectivity is consistent with results from studies using DTI (Persson et al., 2006; Madden et al., 2007) and may reflect attempted compensation in response to reduced connectivity.

\subsection{Implications for Cognitive Aging Theories}

Our findings are consistent with a view that executive functional systems are affected by clinically silent pathologies independent of chronological age (Jagust, 2013). Our fC results in particular appear generally consistent with the brain maintenance theory, which holds that successful cognition in older adults is most closely tied to minimization of brain changes and pathology (Nyberg et al., 2012). In-keeping with this possibility, we found that the same functional pattern of fronto-visual fC was positively correlated with working memory 
accuracy in both younger and older adults but was linked with lower levels of AD pathology in older adults.

Our results may also be in-keeping with aspects of brain dysfunction theories such as efficiency or dedifferentiation theory (Baltes and Lindenberger, 1997; Zarahn et al., 2007). In particular, IDLPFC response magnitudes were associated with longer RT and higher pathology in the older group. The IDLPFC is thought to contribute to proactive control processes that are intended to deal with upcoming conflict and become impaired in older age (Braver, 2012). It may be the case that clinically silent AD and vascular pathologies could contribute to age-related declines in proactive control processes by increasing IDLPFC demands to process noisy information (Salthouse and Lichty, 1985) and/or by reducing its communication with other hubs of the control network.

\subsection{Limitations of Present Study}

Although our results suggest that clinically silent pathologies are associated with frontal hyper-response magnitude and hypo-connectivity, they stop short of assessing a potentially causative relationship between these deleterious activation patterns. For example, the neuronal excitatory-toxicity hypothesis holds that excessive synaptic activity activation leads to a progressive neurodegenerative cascade (Palop and Mucke, 2010). It is thus possible that the high IDLPFC response we observed may contribute to the reduced connectivity between this region and its functionally connected network.

This possibility should be tested in future longitudinal work integrating DTI measures. Integration of DTI and related measures of WM microstructural properties would also be relevant given their association with BOLD response (Madden et al., 2007; Zhu et al., 2015) and more recently with AD pathology (Gold et al., 2014; Molinuevo et al., 2014). Integration of DTI and related imaging measures would thus be likely to boost the modest levels of explained variance in relationships between clinically silent pathology and taskrelevant functional activation patterns we observed. Further, in the context of a longitudinal design, integrating measures of WM microstructural properties would be relevant to testing the theory that degeneration of myelin may contribute to accrual of AD pathology (Bartzokis, 2011). Several potential caveats associated with our hypothesis-driven approach are also worth noting. While our approach identified performance-activation relationships in fronto-visual regions, potential relationships in other brain regions may have been missed. In addition, our ROI masks were generated to surround functional peaks (from the voxelwise ICA) in portions of the IDLPFC and bilateral LOC regions. A different approach would be to generate ROI masks of entire functional activation clusters. In our study, a cluster-based approach would have yielded ROI masks encompassing large activation clusters extending well-beyond the anatomical boundaries of our IDLPFC and LOC ROIs. We adopted the peak-based ROI approach to test hypotheses about activation patterns within these specific regions but note that this approach is statistically more liberal than the cluster-based approach, in which fMRI signals are averaged across larger numbers of voxels with more moderate statistical values. 


\subsection{Conclusion}

Our results suggest that 'healthy' cognitive aging appears to be associated with maintained fronto-visual networks supporting executive control processes. However, clinically silent AD and vascular pathologies are associated with alteration of the functioning of brain circuits used by $\mathrm{CN}$ older adults during executive processes. Specifically, preclinical $\mathrm{AD}$ and vascular pathologies appear to disrupt widespread executive control systems and may contribute to dedifferentation of frontal hubs within this network.

\section{Acknowledgments}

This study was supported by the National Institute on Aging and National Center for Advancing Translational Sciences of the National Institutes of Health (grant numbers RO1AG033036, P30AG028383, P01AG030128, TL1TR000115). In addition, JQT is supported by P30AG10124 and U01AG24904, and LMS is supported by U01AG24904. The authors thank Beverly Meacham for conducting some of the MRI scans and Dr. Gregory Jicha for performing some of the lumbar CSF draws.

\section{References}

Baltes PB, Lindenberger U. Emergence of a powerful connection between sensory and cognitive functions across the adult life span: a new window to the study of cognitive aging? Psychol Aging. 1997; 12:12-21. [PubMed: 9100264]

Bartzokis G. Alzheimer's disease as homeostatic responses to age-related myelin breakdown. Neurobiol Aging. 2011; 32:1341-1371. [PubMed: 19775776]

Beckmann CF, Smith SM. Tensorial extensions of independent component analysis for multisubject FMRI analysis. Neuroimage. 2005; 25:294-311. [PubMed: 15734364]

Bell-McGinty S, Podell K, Franzen M, Baird AD, Williams MJ. Standard measures of executive function in predicting instrumental activities of daily living in older adults. Int J Geriatr Psychiatry. 2002; 17:828-834. [PubMed: 12221656]

Blacker D, Lee H, Muzikansky A, Martin EC, Tanzi R, McArdle JJ, Moss M, Albert M. Neuropsychological measures in normal individuals that predict subsequent cognitive decline. Arch Neurol. 2007; 64:862-871. [PubMed: 17562935]

Braver TS. The variable nature of cognitive control: a dual mechanisms framework. Trends Cogn Sci. 2012; 16:106-113. [PubMed: 22245618]

Breteler MM, van Swieten JC, Bots ML, Grobbee DE, Claus JJ, van den Hout JH, van Harskamp F, Tanghe HL, de Jong PT, van Gijn J, et al. Cerebral white matter lesions, vascular risk factors, and cognitive function in a population-based study: the Rotterdam Study. Neurology. 1994; 44:12461252. [PubMed: 8035924]

Brickman AM, Guzman VA, Gonzalez-Castellon M, Razlighi Q, Gu Y, Narkhede A, Janicki S, Ichise M, Stern Y, Manly JJ, Schupf N, Marshall RS. Cerebral autoregulation, beta amyloid, and white matter hyperintensities are interrelated. Neurosci Lett. 2015; 592:54-58. [PubMed: 25748319]

Cirrito JR, Yamada KA, Finn MB, Sloviter RS, Bales KR, May PC, Schoepp DD, Paul SM, Mennerick S, Holtzman DM. Synaptic activity regulates interstitial fluid amyloid-beta levels in vivo. Neuron. 2005; 48:913-922. [PubMed: 16364896]

Drag LL, Bieliauskas LA. Contemporary review 2009: cognitive aging. J Geriatr Psychiatry Neurol. 2010; 23:75-93. [PubMed: 20101069]

Elman JA, Oh H, Madison CM, Baker SL, Vogel JW, Marks SM, Crowley S, O'Neil JP, Jagust WJ. Neural compensation in older people with brain amyloid-beta deposition. Nat Neurosci. 2014; 17:1316-1318. [PubMed: 25217827]

Fagan AM, Roe CM, Xiong C, Mintun MA, Morris JC, Holtzman DM. Cerebrospinal fluid tau/betaamyloid(42) ratio as a prediction of cognitive decline in nondemented older adults. Arch Neurol. 2007; 64:343-349. [PubMed: 17210801]

Gold BT, Zhu Z, Brown CA, Andersen AH, LaDu MJ, Tai L, Jicha GA, Kryscio RJ, Estus S, Nelson PT, Scheff SW, Abner E, Schmitt FA, Van Eldik LJ, Smith CD. White matter integrity is associated 
with cerebrospinal fluid markers of Alzheimer's disease in normal adults. Neurobiol Aging. 2014; 35:2263-2271. [PubMed: 24866404]

Gordon BA, Zacks JM, Blazey T, Benzinger TL, Morris JC, Fagan AM, Holtzman DM, Balota DA. Task-evoked fMRI changes in attention networks are associated with preclinical Alzheimer's disease biomarkers. Neurobiol Aging. 2015; 36:1771-1779. [PubMed: 25708908]

Grady C. The cognitive neuroscience of ageing. Nat Rev Neurosci. 2012; 13:491-505. [PubMed: 22714020]

Grill-Spector K, Kourtzi Z, Kanwisher N. The lateral occipital complex and its role in object recognition. Vision Res. 2001; 41:1409-1422. [PubMed: 11322983]

Gurol ME, Viswanathan A, Gidicsin C, Hedden T, Martinez-Ramirez S, Dumas A, Vashkevich A, Ayres AM, Auriel E, van Etten E, Becker A, Carmasin J, Schwab K, Rosand J, Johnson KA, Greenberg SM. Cerebral amyloid angiopathy burden associated with leukoaraiosis: a positron emission tomography/magnetic resonance imaging study. Ann Neurol. 2013; 73:529-536. [PubMed: 23424091]

Hakun JG, Zhu Z, Johnson NF, Gold BT. Evidence for reduced efficiency and successful compensation in older adults during task switching. Cortex. 2015; 64:352-362. [PubMed: 25614233]

Harada CN, Natelson Love MC, Triebel KL. Normal cognitive aging. Clinics in geriatric medicine. 2013; 29:737-752. [PubMed: 24094294]

Hardy J, Selkoe DJ. The amyloid hypothesis of Alzheimer's disease: progress and problems on the road to therapeutics. Science. 2002; 297:353-356. [PubMed: 12130773]

Hedden T, Park DC. Contributions of source and inhibitory mechanisms to age-related retroactive interference in verbal working memory. J Exp Psychol Gen. 2003; 132:93-112. [PubMed: 12656299]

Iqbal K, Liu F, Gong CX, Alonso Adel C, Grundke-Iqbal I. Mechanisms of tau-induced neurodegeneration. Acta Neuropathol. 2009; 118:53-69. [PubMed: 19184068]

Jagust W. Vulnerable neural systems and the borderland of brain aging and neurodegeneration. Neuron. 2013; 77:219-234. [PubMed: 23352159]

Jenkinson M, Beckmann CF, Behrens TE, Woolrich MW, Smith SM. Fsl. Neuroimage. 2012; 62:782790. [PubMed: 21979382]

Kane MJ, Engle RW. The role of prefrontal cortex in working-memory capacity, executive attention, and general fluid intelligence: an individual-differences perspective. Psychon Bull Rev. 2002; 9:637-671. [PubMed: 12613671]

Lazarov O, Lee M, Peterson DA, Sisodia SS. Evidence that synaptically released beta-amyloid accumulates as extracellular deposits in the hippocampus of transgenic mice. J Neurosci. 2002; 22:9785-9793. [PubMed: 12427834]

Lockhart SN, Luck SJ, Geng J, Beckett L, Disbrow EA, Carmichael O, DeCarli C. White matter hyperintensities among older adults are associated with futile increase in frontal activation and functional connectivity during spatial search. PLoS One. 2015; 10:e0122445. [PubMed: 25793922]

Madden DJ, Spaniol J, Whiting WL, Bucur B, Provenzale JM, Cabeza R, White LE, Huettel SA. Adult age differences in the functional neuroanatomy of visual attention: a combined fMRI and DTI study. Neurobiol Aging. 2007; 28:459-476. [PubMed: 16500004]

Mayda AB, Westphal A, Carter CS, DeCarli C. Late life cognitive control deficits are accentuated by white matter disease burden. Brain. 2011; 134:1673-1683. [PubMed: 21482547]

Miller EK, Cohen JD. An integrative theory of prefrontal cortex function. Annu Rev Neurosci. 2001; 24:167-202. [PubMed: 11283309]

Miyake A, Friedman NP, Emerson MJ, Witzki AH, Howerter A, Wager TD. The unity and diversity of executive functions and their contributions to complex "Frontal Lobe" tasks: a latent variable analysis. Cogn Psychol. 2000; 41:49-100. [PubMed: 10945922]

Molinuevo JL, Ripolles P, Simo M, Llado A, Olives J, Balasa M, Antonell A, Rodriguez-Fornells A, Rami L. White matter changes in preclinical Alzheimer's disease: a magnetic resonance imagingdiffusion tensor imaging study on cognitively normal older people with positive amyloid beta protein 42 levels. Neurobiol Aging. 2014; 35:2671-2680. [PubMed: 25002037] 
Mormino EC, Brandel MG, Madison CM, Marks S, Baker SL, Jagust WJ. Abeta Deposition in aging is associated with increases in brain activation during successful memory encoding. Cereb Cortex. 2012; 22:1813-1823. [PubMed: 21945849]

Morris JC, Weintraub S, Chui HC, Cummings J, Decarli C, Ferris S, Foster NL, Galasko D, GraffRadford N, Peskind ER, Beekly D, Ramos EM, Kukull WA. The Uniform Data Set (UDS): clinical and cognitive variables and descriptive data from Alzheimer Disease Centers. Alzheimer Dis Assoc Disord. 2006; 20:210-216. [PubMed: 17132964]

Niendam TA, Laird AR, Ray KL, Dean YM, Glahn DC, Carter CS. Meta-analytic evidence for a superordinate cognitive control network subserving diverse executive functions. Cogn Affect Behav Neurosci. 2012; 12:241-268. [PubMed: 22282036]

Nordahl CW, Ranganath C, Yonelinas AP, Decarli C, Fletcher E, Jagust WJ. White matter changes compromise prefrontal cortex function in healthy elderly individuals. J Cogn Neurosci. 2006; 18:418-429. [PubMed: 16513006]

Nyberg L, Lovden M, Riklund K, Lindenberger U, Backman L. Memory aging and brain maintenance. Trends Cogn Sci. 2012; 16:292-305. [PubMed: 22542563]

Oh H, Jagust WJ. Frontotemporal network connectivity during memory encoding is increased with aging and disrupted by beta-amyloid. J Neurosci. 2013; 33:18425-18437. [PubMed: 24259567]

Oh H, Steffener J, Razlighi QR, Habeck C, Liu D, Gazes Y, Janicki S, Stern Y. Abeta-related hyperactivation in frontoparietal control regions in cognitively normal elderly. Neurobiol Aging. 2015; 36:3247-3254. [PubMed: 26382734]

Palop JJ, Mucke L. Amyloid-beta-induced neuronal dysfunction in Alzheimer's disease: from synapses toward neural networks. Nat Neurosci. 2010; 13:812-818. [PubMed: 20581818]

Pearson RC, Powell TP. The neuroanatomy of Alzheimer's disease. Reviews in the neurosciences. 1989; 2:101-122. [PubMed: 21561251]

Persson J, Nyberg L, Lind J, Larsson A, Nilsson LG, Ingvar M, Buckner RL. Structure-function correlates of cognitive decline in aging. Cereb Cortex. 2006; 16:907-915. [PubMed: 16162855]

Reed BR, Eberling JL, Mungas D, Weiner M, Kramer JH, Jagust WJ. Effects of white matter lesions and lacunes on cortical function. Arch Neurol. 2004; 61:1545-1550. [PubMed: 15477508]

Rissman J, Gazzaley A, D'Esposito M. Measuring functional connectivity during distinct stages of a cognitive task. Neuroimage. 2004; 23:752-763. [PubMed: 15488425]

Salthouse TA, Lichty W. Tests of the neural noise hypothesis of age-related cognitive change. $\mathbf{J}$ Gerontol. 1985; 40:443-450. [PubMed: 4008879]

Sander MC, Lindenberger U, Werkle-Bergner M. Lifespan age differences in working memory: a twocomponent framework. Neurosci Biobehav Rev. 2012; 36:2007-2033. [PubMed: 22771333]

Schaie, KW. Intellectual Development in Adulthood: The Seattle Longitudinal Study. Cambridge: Cambridge University Press; 1996.

Schmitt FA, Nelson PT, Abner E, Scheff S, Jicha GA, Smith C, Cooper G, Mendiondo M, Danner DD, Van Eldik LJ, Caban-Holt A, Lovell MA, Kryscio RJ. University of Kentucky Sanders-Brown healthy brain aging volunteers: donor characteristics, procedures and neuropathology. Curr Alzheimer Res. 2012; 9:724-733. [PubMed: 22471862]

Scott JA, Braskie MN, Tosun D, Maillard P, Thompson PM, Weiner M, DeCarli C, Carmichael OT. Cerebral amyloid is associated with greater white-matter hyperintensity accrual in cognitively normal older adults. Neurobiol Aging. 2016; 48:48-52. [PubMed: 27639120]

Shaw LM, Vanderstichele H, Knapik-Czajka M, Clark CM, Aisen PS, Petersen RC, Blennow K, Soares H, Simon A, Lewczuk P, Dean R, Siemers E, Potter W, Lee VM, Trojanowski JQ. Cerebrospinal fluid biomarker signature in Alzheimer's disease neuroimaging initiative subjects. Ann Neurol. 2009; 65:403-413. [PubMed: 19296504]

Smith CD, Johnson ES, Van Eldik LJ, Jicha GA, Schmitt FA, Nelson PT, Kryscio RJ, Murphy RR, Wellnitz CV. Peripheral (deep) but not periventricular MRI white matter hyperintensities are increased in clinical vascular dementia compared to Alzheimer's disease. Brain and behavior. 2016; 6:e00438. [PubMed: 26925303]

Smith SM, Jenkinson M, Woolrich MW, Beckmann CF, Behrens TE, Johansen-Berg H, Bannister PR, De Luca M, Drobnjak I, Flitney DE, Niazy RK, Saunders J, Vickers J, Zhang Y, De Stefano N, 
Brady JM, Matthews PM. Advances in functional and structural MR image analysis and implementation as FSL. Neuroimage. 2004; 23(Suppl 1):S208-219. [PubMed: 15501092]

Snodgrass JG, Vanderwart M. A standardized set of 260 pictures: norms for name agreement, image agreement, familiarity, and visual complexity. Journal of experimental psychology Human learning and memory. 1980; 6:174-215. [PubMed: 7373248]

Sperling RA, Laviolette PS, O'Keefe K, O'Brien J, Rentz DM, Pihlajamaki M, Marshall G, Hyman BT, Selkoe DJ, Hedden T, Buckner RL, Becker JA, Johnson KA. Amyloid deposition is associated with impaired default network function in older persons without dementia. Neuron. 2009; 63:178188. [PubMed: 19640477]

Venkatraman VK, Aizenstein H, Guralnik J, Newman AB, Glynn NW, Taylor C, Studenski S, Launer L, Pahor M, Williamson J, Rosano C. Executive control function, brain activation and white matter hyperintensities in older adults. Neuroimage. 2010; 49:3436-3442. [PubMed: 19922803]

Vos SJ, Xiong C, Visser PJ, Jasielec MS, Hassenstab J, Grant EA, Cairns NJ, Morris JC, Holtzman DM, Fagan AM. Preclinical Alzheimer's disease and its outcome: a longitudinal cohort study. Lancet Neurol. 2013; 12:957-965. [PubMed: 24012374]

Weintraub S, Salmon D, Mercaldo N, Ferris S, Graff-Radford NR, Chui H, Cummings J, DeCarli C, Foster NL, Galasko D, Peskind E, Dietrich W, Beekly DL, Kukull WA, Morris JC. The Alzheimer's Disease Centers' Uniform Data Set (UDS): the neuropsychologic test battery. Alzheimer Dis Assoc Disord. 2009; 23:91-101. [PubMed: 19474567]

Zarahn E, Rakitin B, Abela D, Flynn J, Stern Y. Age-related changes in brain activation during a delayed item recognition task. Neurobiol Aging. 2007; 28:784-798. [PubMed: 16621168]

Zhu Z, Johnson NF, Kim C, Gold BT. Reduced frontal cortex efficiency is associated with lower white matter integrity in aging. Cereb Cortex. 2015; 25:138-146. [PubMed: 23960206]

Zlokovic BV. Neurovascular mechanisms of Alzheimer's neurodegeneration. Trends Neurosci. 2005; 28:202-208. [PubMed: 15808355] 


\section{Highlights}

The impact of preclinical pathologies on executive brain systems was explored

Older and younger adults completed a functional neuroimaging (fMRI) paradigm

Alzheimer's (AD) pathology and white matter hyperintensities (WMH) were computed AD pathology and WMH negatively affected fMRI patterns in older adults 


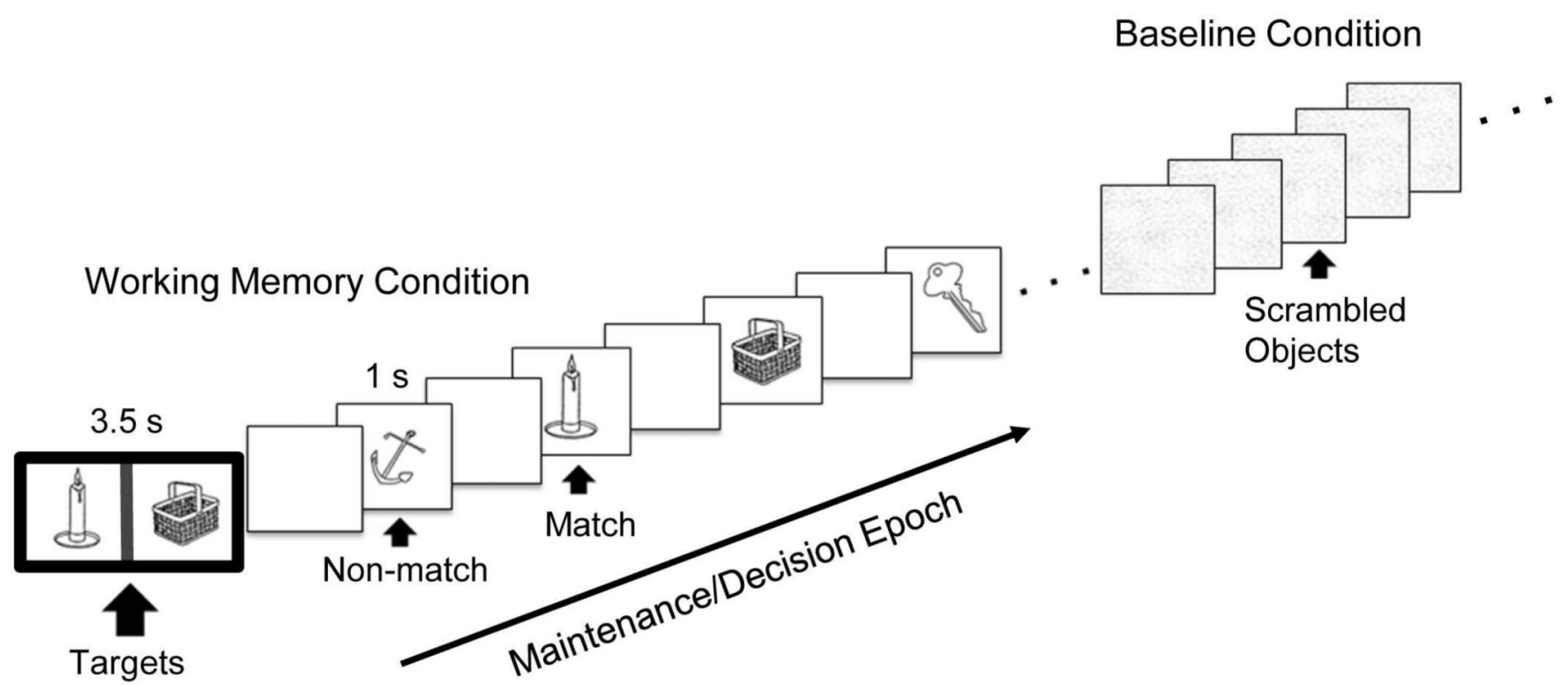

Figure 1.

A schematic of the working memory paradigm. Task blocks involved presentation of two target images surrounded by a dark border followed by 'test' images (match or non-match). Blank screens between images represent temporal jittering. Baseline (control) blocks involved viewing scrambled images. Analyses focused on the 'maintenance/decision' epoch of blocks, as indicated by the arrow. 
A
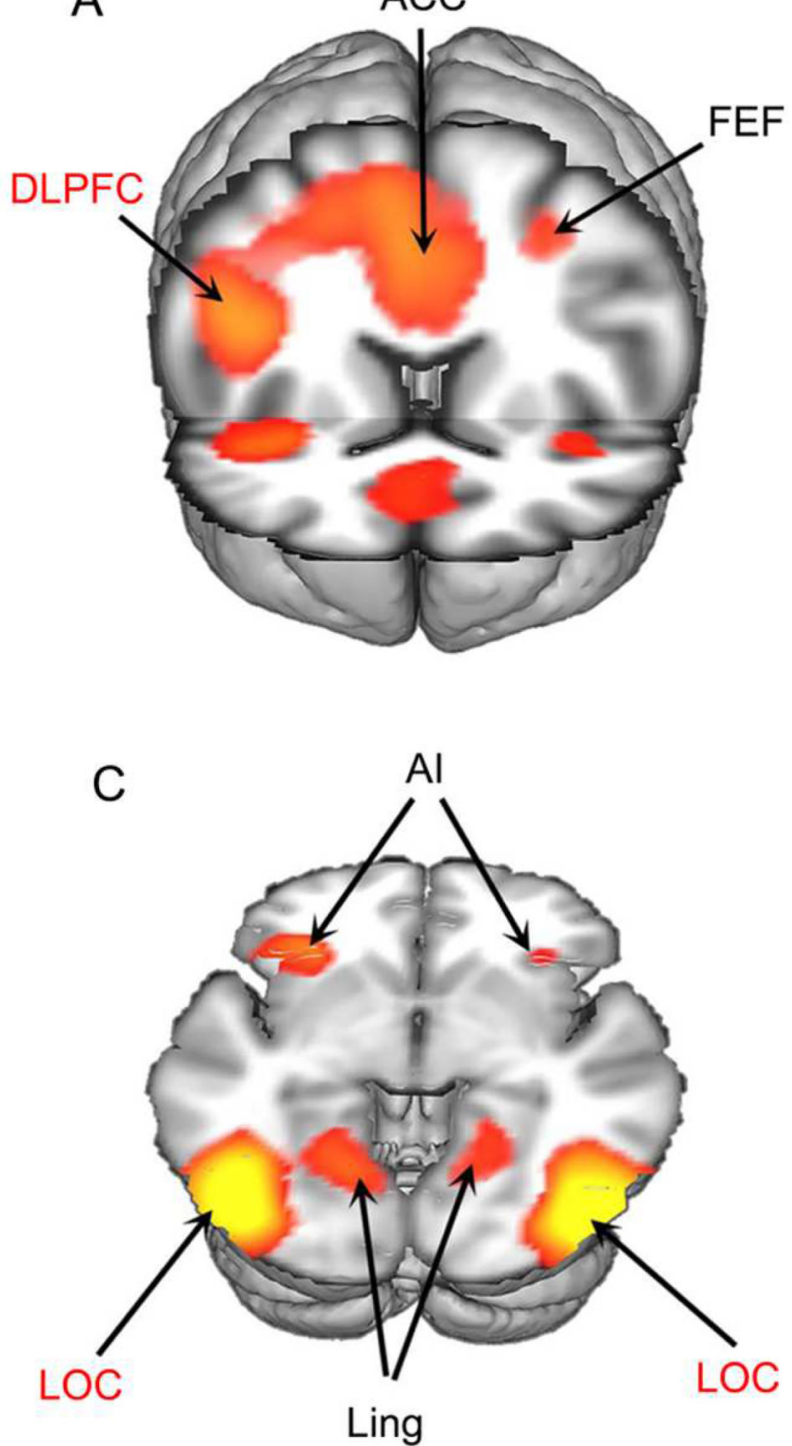

Figure 2.

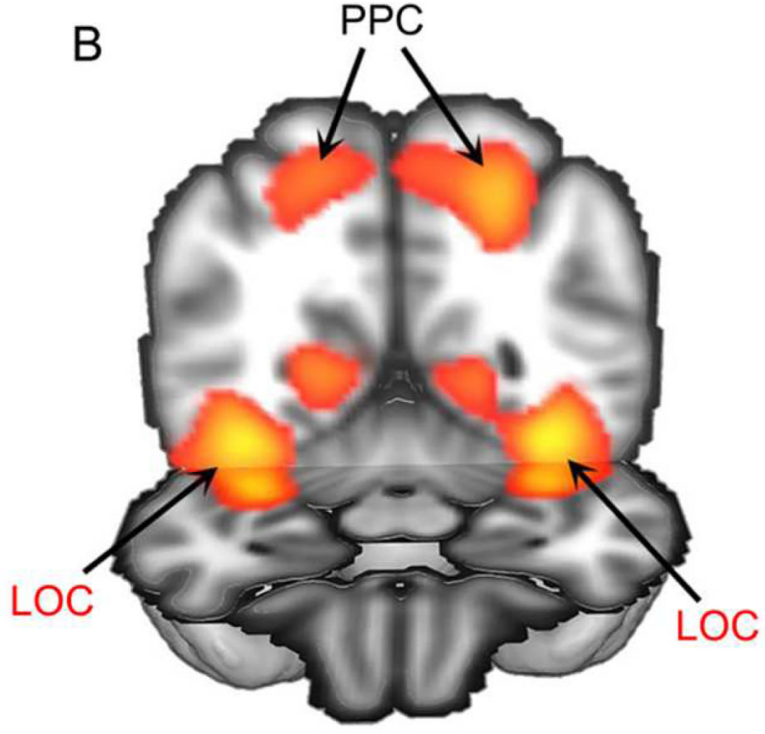

$\mathrm{R}$

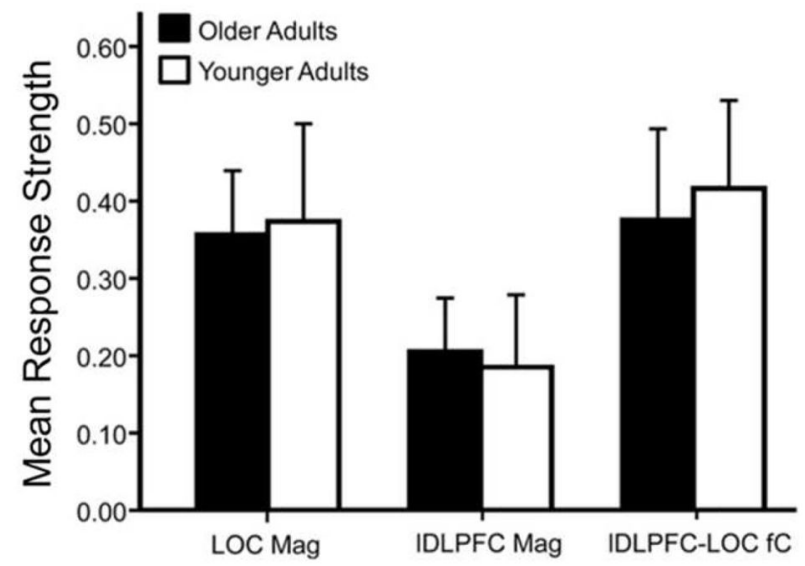

ICA Results and ROI Mean Values. The main ICA component is shown in coronal (A-B) and axial (C) views. Regions selected for a priori analyses are in red font. ACC: anterior cingulate cortex; FEF: frontal eye fields; AI: anterior insula; DLPFC: dorsolateral prefrontal cortex; LOC: lateral occipital cortex; PPC: posterior parietal cortex; Ling: lingual gyrus. The bar chart summarizes group mean response strengths in the selected ROIs (D). Values for BOLD magnitude represent mean percent signal change between task and baseline in LOC (LOC mag) and IDLPFC (IDLPFC mag). Values for fC represent connectivity scores (IDLPFC-LOC fC). 
A

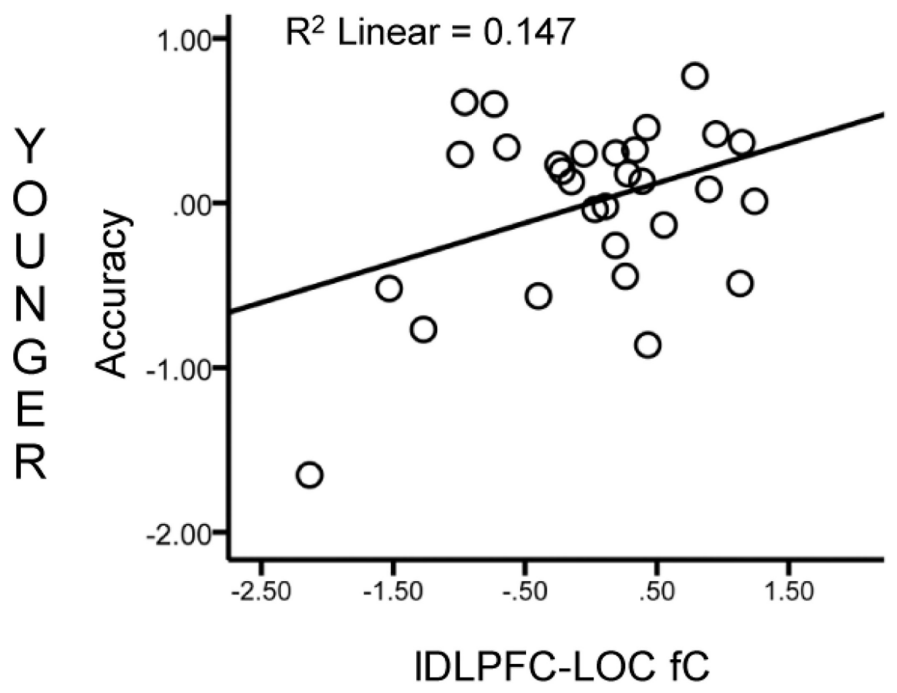

C

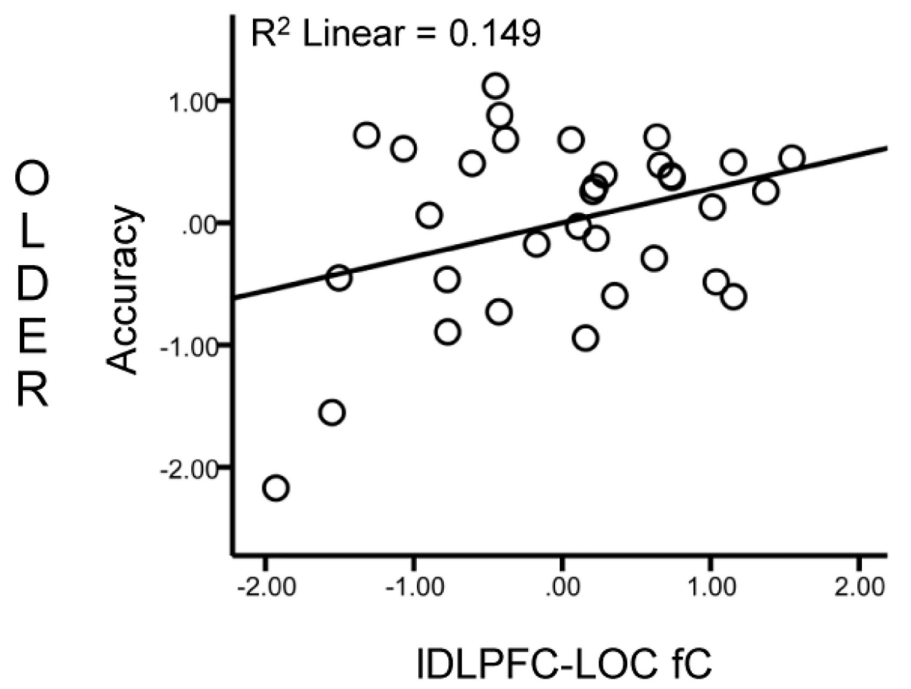

B
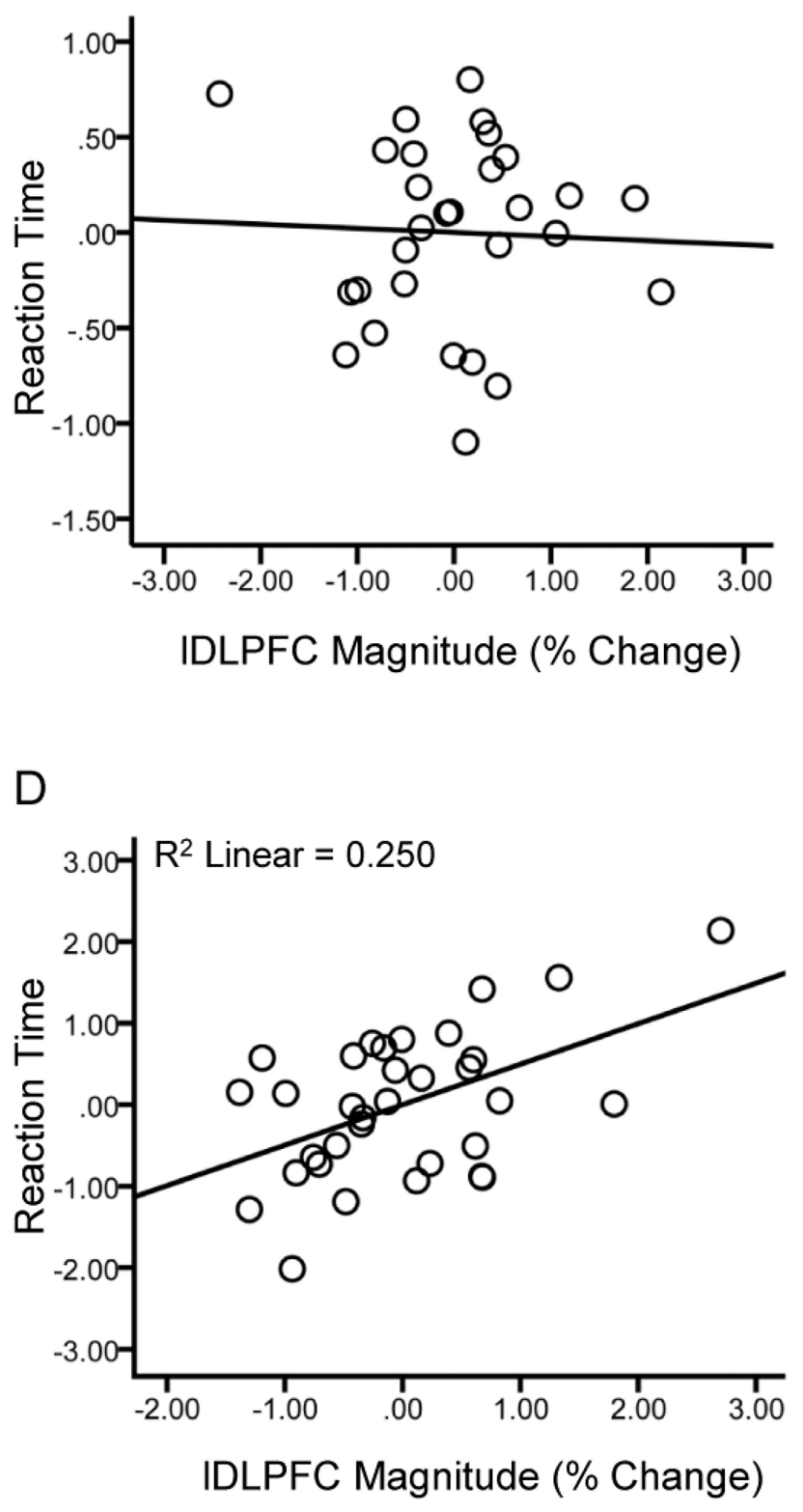

Figure 3.

Relationships between functional activation patterns and performance. Scatter plots of IDLPFC-LOC fC scores against accuracy (A) and IDLPFC BOLD magnitude against RT (B). Values are standardized residuals after controlling for age, sex and education.

Significant relationships are shown with lines representing the linear best fit. Results are shown for younger adults (top) and older adults (bottom). 


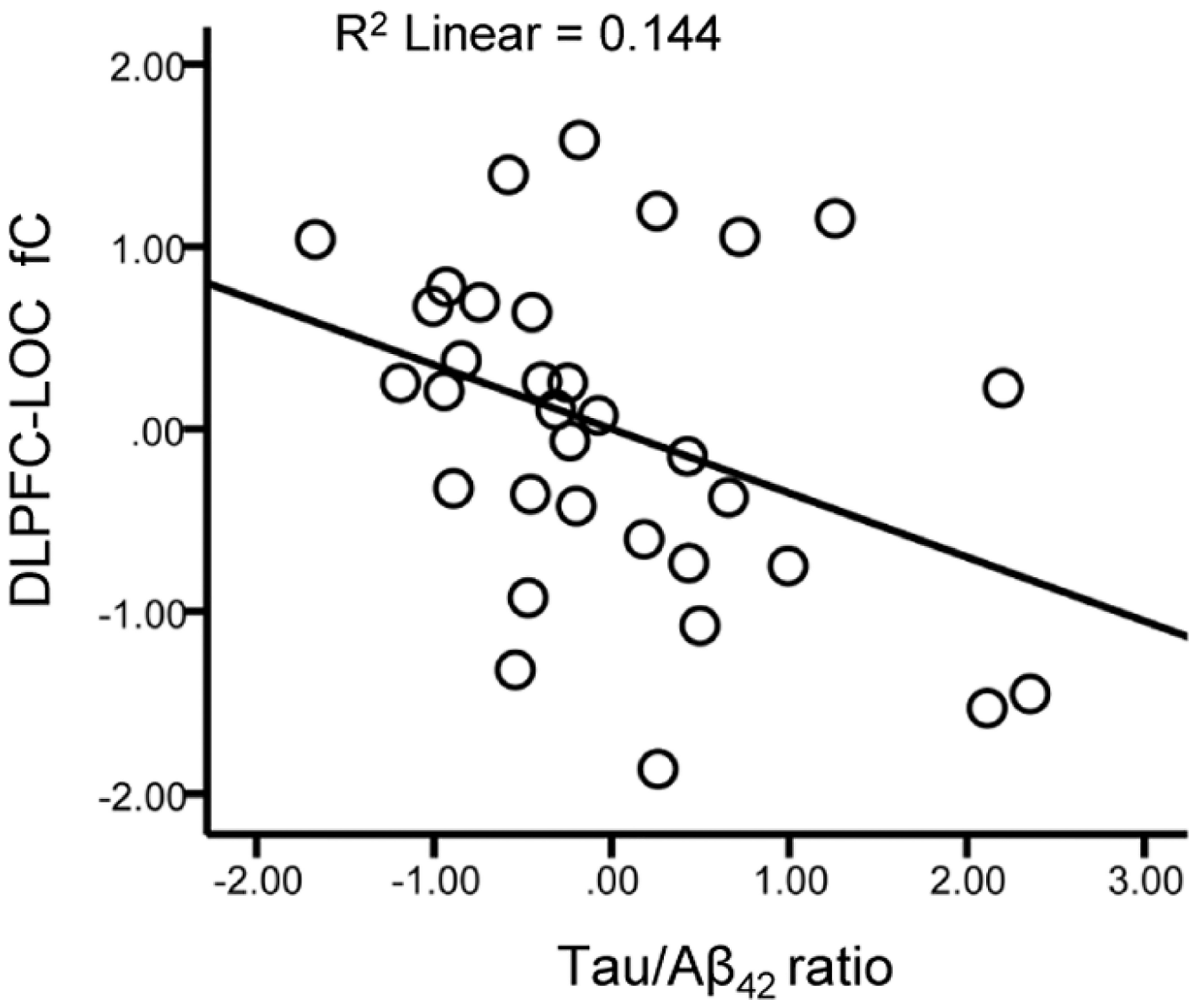

Figure 4.

Relationship between CSF markers and fronto-visual fC. Scatter plot of CSF t-tau/A $\beta_{42}$ ratio scores against IDLPFC-LOC fC scores. Values are standardized residuals after controlling for age, sex and education. 
A

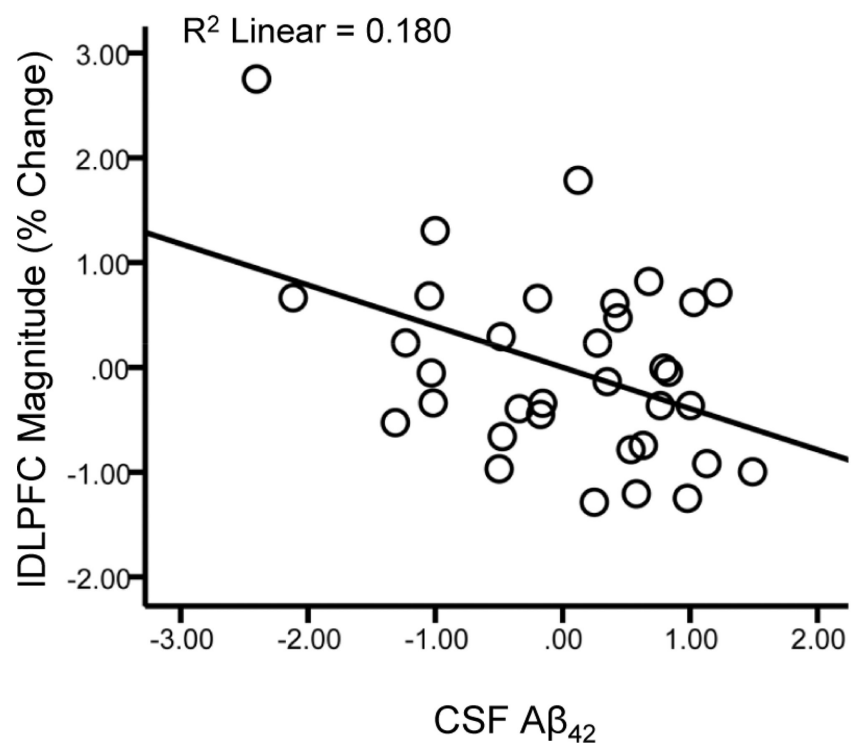

B

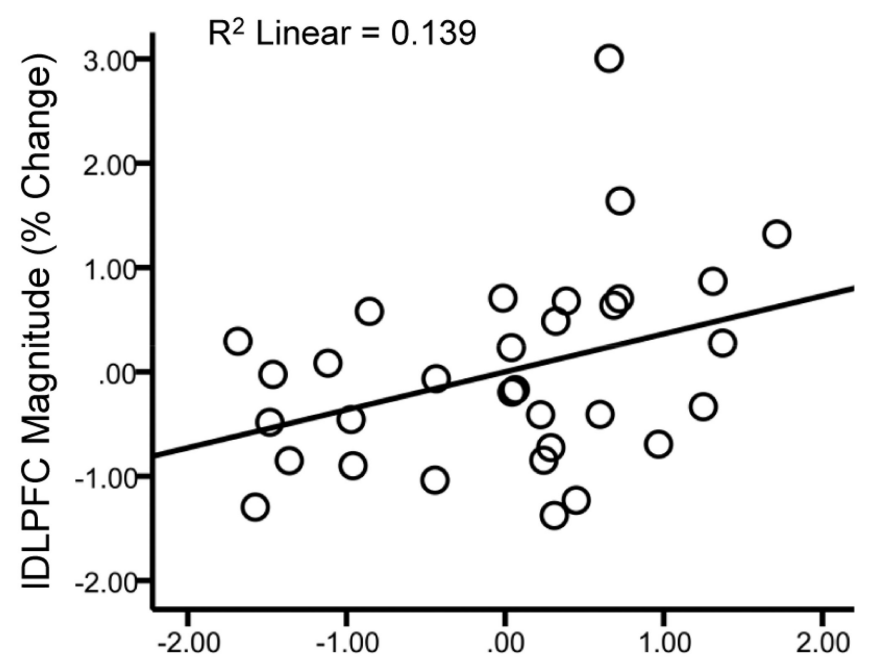

Frontal WMH Volume

Figure 5.

Relationships between left DLPFC response magnitude and pathology markers. Scatter plots of IDLPFC magnitude against CSF A $\beta_{42}(\mathrm{~A})$ and frontal WMH volume (B). Values are standardized residuals after controlling for age, sex and education. 


\section{Table 1}

Demographics, risk factors and behavioral scores

\begin{tabular}{llll}
\hline & $\begin{array}{l}\text { Younger adults } \\
(\mathbf{n = 2 9})\end{array}$ & $\begin{array}{l}\text { Older adults } \\
(\mathbf{n = 3 4})\end{array}$ & p-value \\
\hline Age (Years) & $24.14(3.9)$ & $77.03(7.0)$ & $<.001$ \\
M/F & $12 / 17$ & $15 / 19$ & .748 \\
Education (Years) & $16.6(2.1)$ & $16.6(2.4)$ & .928 \\
APOE $\varepsilon 4$ & - & $24 \%$ & - \\
BMI & - & $26.4(4.7)$ & - \\
Systolic BP & - & $137.2(12.6)$ & - \\
Diastolic BP & - & $76.6(10.7)$ & - \\
Corrected Recognition & $0.94(0.07)$ & $0.86(0.10)$ & $<.001$ \\
Reaction Time (ms) & $543(62)$ & $723(86)$ & $<.001$ \\
\hline
\end{tabular}

M/F: male/female; APOE $\varepsilon 4$ : percentage of older adults with one or more APOE $\varepsilon 4$ alleles; BMI: body mass index; BP: blood pressure. Values represent means (standard deviations) for continuous variables and either ratio (M/F) or percentages (APOE $\varepsilon 4$ ) for categorical variables. Data was missing for APOE $\varepsilon 4$ (26\%), BMI (17\%) and BP (17\%). P-values were calculated from independent samples t-tests, except for M/F, which was calculated from a Chi-square test. 


\section{Table 2}

\section{Peak coordinates for selected IC}

\begin{tabular}{lcccc} 
& Peak & MNI & MNI & MNI \\
& Z-value & $\mathbf{X}$ & $\mathbf{Y}$ & $\mathbf{Z}$ \\
\hline Left dorsolateral prefrontal cortex & 3.35 & -46 & 20 & 22 \\
Right lateral occipital cortex & 5.76 & 40 & -86 & -4 \\
Left lateral occipital cortex & 5.72 & -40 & -88 & -4 \\
Left lingual gyrus & 5.89 & -8 & -76 & 10 \\
Right lingual gyrus & 5.60 & 10 & -72 & 12 \\
Anterior cingulate cortex & 5.17 & -4 & 12 & 48 \\
Left posterior parietal cortex & 4.36 & -26 & -54 & 50 \\
Right posterior parietal cortex & 3.40 & 24 & -52 & 54 \\
Left frontal eye fields & 4.66 & -26 & -4 & 48 \\
Left anterior insula & 3.66 & -34 & 26 & -2 \\
Right frontal eye fields & 3.59 & 30 & -2 & 46 \\
Right anterior insula & 2.56 & 36 & 24 & 2 \\
\hline
\end{tabular}

Peak coordinates from ICA corresponding to the main working memory task component. Peak Z-values is the Z-score of the MNI-coordinate for the selected cluster. Regions used in hypothesis-driven analyses are listed first. 
Table 3

CSF protein concentrations, WMH volumes and Vascular Factors

\begin{tabular}{|c|c|c|}
\hline & Mean & SD \\
\hline $\mathrm{A} \beta_{42}(\mathrm{pg} / \mathrm{mL})$ & 272.9 & 81.0 \\
\hline $\mathrm{t}$-tau $(\mathrm{pg} / \mathrm{mL})$ & 56.06 & 16.1 \\
\hline p-tau $181(\mathrm{pg} / \mathrm{mL})$ & 23.5 & 7.49 \\
\hline $\mathrm{t}$-tau/A $\beta_{42}$ ratio & 0.234 & 0.137 \\
\hline whole-brain WMHs & 0.44 & 0.24 \\
\hline frontal WMHs & 0.24 & 0.19 \\
\hline
\end{tabular}

Aß42: beta-amyloid (1-42); t-tau: total tau; p-tau181: phosphorylated tau. WMHs; white matter hyperintensity volume (\% of ICV). 